\title{
Geographical Mechanisms of Regional Differentiation and Evolution of Pronunciation and Singing Tune Under the New Mobility Paradigm__A Case Study of Ten Local Operas in China
}

\author{
Qin $\mathrm{Liu}^{1}$, Kai Xu${ }^{1}$, Xiaojun Yang ${ }^{1,2,3 *}$ \\ ${ }^{1}$ Xi'an International Studies University, Tourism of College and Institute of Human Geography, Xi'an, Shaanxi \\ Province,710128, China \\ ${ }^{2}$ Shaanxi Tourism Research Institute, Xi'an, Shaanxi province, 710128, China \\ ${ }^{3}$ Innovative Team of Urban-Rural Space Integration Development; \\ *Corresponding author's e-mail: yangxiaojun@xisu.edu.cn
}

\begin{abstract}
Opera is a traditional culture integrating mobility and immobility. Based on the perspective of new flow paradigm, this paper adopts the spatial analysis method to analyse the regional distribution characteristics and formation mechanism of ten operas. The research shows that: (1) Based on the Qinling Mountains-Huaihe River line, Henan Opera, Jin Opera and Hebei clapper Opera are distributed in circular layers with the origin as the center, Shaanxi Opera is mainly distributed in the west, and Ping opera is distributed in a double-center structure, forming a distribution structure with dialects as the link. The southern operas are affected by the terrain. Huangmei Opera, Cantonese Opera and Sichuan Opera are distributed in circles, while Shaoxing Opera and Kun Opera are distributed in points. (2) Although the northern operas originated earlier, the stability of singing is poor and the cultural region is shrinking seriously. The southern operas originated late, but influenced by the terrain and have obvious internal circulation, the singing is more stable and the space contraction is less. (3) From the perspective of the new mobility paradigm, a spatial mobility system with economy as the pulling force, climate and terrain as the pushing force, population flow, daily life as the media, and policy protection dialect features should be formed.
\end{abstract}

Keywords: mobility, top 10 local operas, pronunciation, singing tune, geography, mechanisms

\section{INTRODUCTION}

China has a vast territory and obvious regional differences, forming a unique local opera. Opera is a regional cultural landscape integrating music, dance, literature and art. It is also a typical representative of regional locality. It plays an important role in traditional culture. With the acceleration of urbanization and networking, traditional entertainment is seriously blocked. How to interpret the regional changes of opera from a new perspective and explore the blocking mechanism of opera cultural development in multiple directions is an urgent problem to improve the regional cultural value. As far as the selected articles are concerned, opera research is mainly divided into two categories according to the participating disciplines, musicology and geography. Among them, musicology mainly focuses on the characteristics of singing and dialect pronunciation[1-3]. Geography studies opera from the influence of geographical environment on opera, and reveals the role of geographical environment in the formation of opera culture[4-5].In the interdisciplinary field of the two disciplines, there are more exchanges and integration in the two directions of cultural geography and music geography [6]. For example, geographers Zhang Jian, Wei Qianru and Liu Qiang study Qin opera and Shaoxing opera based on cultural landscape and cultural diffusion[7-9], musicologist Wu Feng studies the cultural ecology of Henan Opera and Luo Jinman's development of Da opera based on the perspective of cultural geography[10], which shows that the exchanges and integration between the two universities in the field of opera research are deepening, However, there are 
relatively few interdisciplinary drama research results based on geography and sociology.

Opera culture has experienced material flow from its emergence to development, and pronunciation and singing, as the core of drama, have also experienced dynamic changes in space. As a novel perspective for scholars to study the mechanism and law of mobility phenomenon from the perspective of space, as well as the experience, difference and multiple social relations generated in the process of mobility, mobility is a way of world operation by interweaving people, places, objects and emotions based on specific background and context[11].

Before the 1970s, western scholars, most notably philosopher Henri Bergson and geographer Walter Christaller, focused on the study of mobility at the level of theoretical cognition. From the 1970s to the late 1990s, mobility, as a new perspective, gradually became the focus of multidisciplinary research. Scholars began to pay attention to mobility phenomenon from the empirical level, and geographers mostly paid attention to the law and efficiency of mobility from the perspective of space science[12]. However, sociology mostly studies mobility based on social attributes such as occupation, income, education and intergenerational, and pays less attention to the spatial differentiation caused by social development. At present, scholars have begun to reflect on mobility itself and pay more attention to the experience and practice generated under the new mobility paradigm, which has created interdisciplinary research methods and perspectives and endowed the mobility phenomenon with richer social and cultural significance[13].

At present, there are abundant research results on liquidity at home and abroad, but less attention is paid to the space-time dimension of liquidity. In many opera studies, the individual research results on the singing characteristics and pronunciation characteristics of opera are relatively fruitful, but there are relatively few achievements to explore the dynamic change law and social and cultural significance of opera from an interdisciplinary perspective, especially from the perspective of mobility, and the research to systematically analyze the regional differentiation between pronunciation and singing is also relatively insufficient.

Therefore, based on the new mobility paradigm, this paper interprets the temporal and spatial evolution law of opera mobility by using GIS mapping, studies the evolution law of pronunciation and singing under dynamic changes. This paper summarizes the regional differentiation phenomenon of drama, explains its internal mechanism with geographical knowledge, aims to deepen the understanding of drama from the dimension of time and space, and use the development law of drama to better preserve the local cultural gene, and realize the protection and development of drama.

\section{REGIONAL DIFFERENTIATION OF LOCAL OPERA PRONUNCIATION AND SINGING}

\subsection{Research methods and case area introduction}

\subsection{1 research method}

First of all, through literature analysis, extensively read papers related to local operas, systematically master the development process of the top ten local operas, and provide a theoretical foundation for this paper. Secondly, through summary, grasp the research trends of the new flow paradigm, and clarify the application of the new mobility paradigm in the field of opera. Based on Aharon's perspective of mobility[14],this paper constructs the spatial flow diagram of drama under the new flow paradigm by referring to the theories of cultural geography and other disciplines (Fig 1). The new mobility paradigm is a new research framework integrated from multiple perspectives. Its essence is to go beyond the perspectives of geographical research and social research, focusing on a series of substantive significance caused by mobility[15]. Opera under the new paradigm is a unity of mobility and immobility. Its spatial mobility motivation is mainly divided into two categories, namely push and pull. Distance and movement play a driving role in the process of flow, on the contrary, the people, things, places, knowledge or information related to opera exist as a pulling force. As the fundamental attribute of material development, mobility is the attribute feature and existence mode of opera existence and development, which can be spread through daily life and immigration. Immobility is the distinguishing feature of different drama landscapes. Its core elements are pronunciation and singing, and regional characteristics are retained through mandatory policies and settlements.

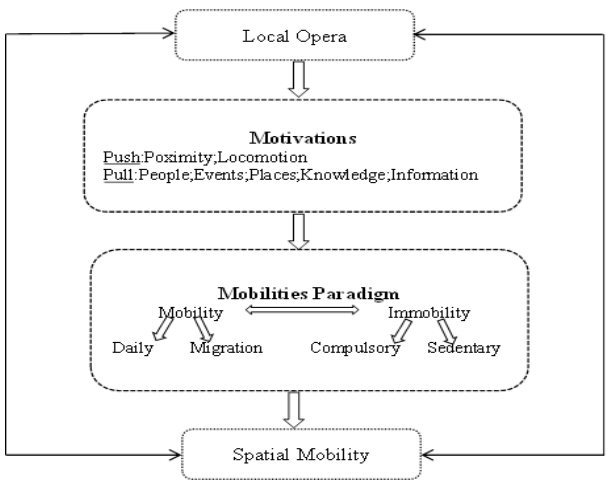

Fig. 1 Spatial mobility diagram of local opera under new mobility paradigm 
Finally, the method combining quantitative analysis and case study is used. By collecting the general situation and spatial data of the selected ten local operas, this paper uses GIS to explain the changes in the pronunciation and singing of operas from the two dimensions of time and space, and predicts the development trend of operas. In order to better retain the local cultural gene, carry forward the traditional culture, highlight the role of geography in cultural development.

\subsubsection{Case area introduction}

Chinese opera culture has a long history and a very rich variety of operas. At present, there is no clear dividing standard for the ten local operas. Therefore, based on two factors of distribution range and active degree, this paper selects five influential local operas in southern and northern regions as research objects. Among the five traditional operas, Beijing opera ranks first, but it is not included because it belongs to the quintessence of China, also known as national opera, and does not belong to local opera. So, the ten local operas finally selected are Shaoxing opera, Henan opera, Ping opera, Huangmei opera, Kun opera, Shaanxi opera, Sichuan opera, Jin opera, Hebei clapper opera and Cantonese opera (Fig 2). Based on the Qinlinghuaihe line, ten local operas can be divided into northern operas and southern operas. Henan opera, Ping opera, Shanxi opera, Jin opera and hebei clapper opera represent the northern operas, while the southern operas are composed of Shaoxing opera, Huangmei Opera, Kun Opera, Sichuan Opera and Cantonese opera. As the wind vane of local opera, the top ten local operas have great regional influence. It is of great significance to analyze the evolution mechanism of local opera.

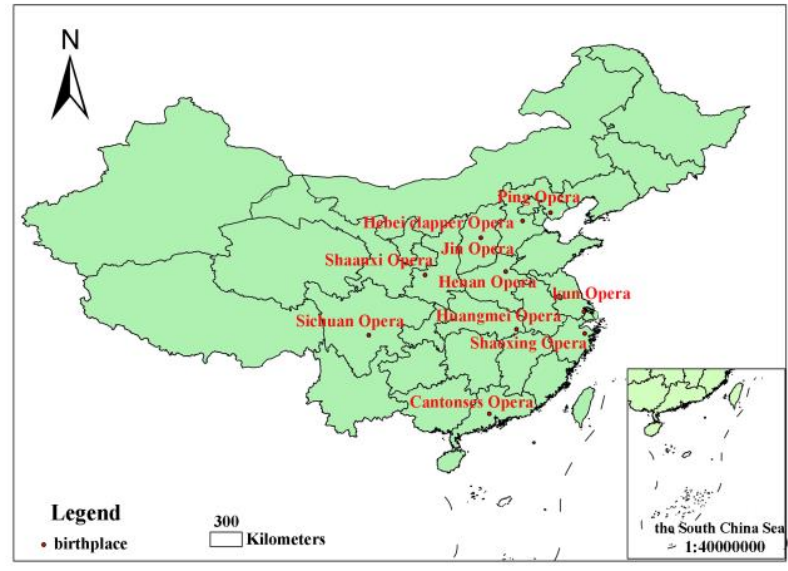

Fig.2 The origin of local operas

\subsection{Spatial distribution structure and relationship of local operas}

Combined with fig 2 and 3, it can be seen that local operas are affected by various factors in the process of spreading from the birthplace to the outside, and finally form the spatial distribution pattern of modern operas: the popular areas of operas are relatively stable, and the distribution structure is relatively diverse. Most operas are distributed in circles centered on the birthplace, but the structure of Ping opera is relatively special. The current distribution pattern of opera is the result of the interaction between different cultural elements in various regions. From the regional distribution, the distribution range of the five northern operas is mainly in the vast areas of the north, and the birthplace and popular range of Southern operas are mainly in the south. From the perspective of distribution structure, opera has four different distribution structures. Some operas are distributed in a circle structure centered on the birthplace and spread to the surrounding neighbors through the floating population. The closer to the core area, the wider the cultural audience. The locality follows the attenuation law of spatial distance, such as Henan opera, Jin opera, Huangmei Opera, Cantonese opera, Hebei clapper opera and Sichuan opera. Some operas are distributed along a certain direction. Shaanxi opera is widely active in the five northwest provinces mainly through West diffusion. It has unique cultural value in the five northwest provinces, which is inseparable from the unique geographical location and social environment of the northwest. In addition, operas are distributed in dots. Kun Opera and Shaoxing opera originated in the Yangtze River Delta. Influenced by the dense river network, operas are distributed in dots. Now they are mainly popular in Jiangsu, Shanghai, Zhejiang, Jiangxi and other places. Moreover, traditional opera presents a dual center structure distribution, and Ping opera is the most typical. In addition to being popular in Beijing, Tianjin and Hebei, Ping opera is also concentrated in the three eastern provinces and is developing around the two central areas [16].

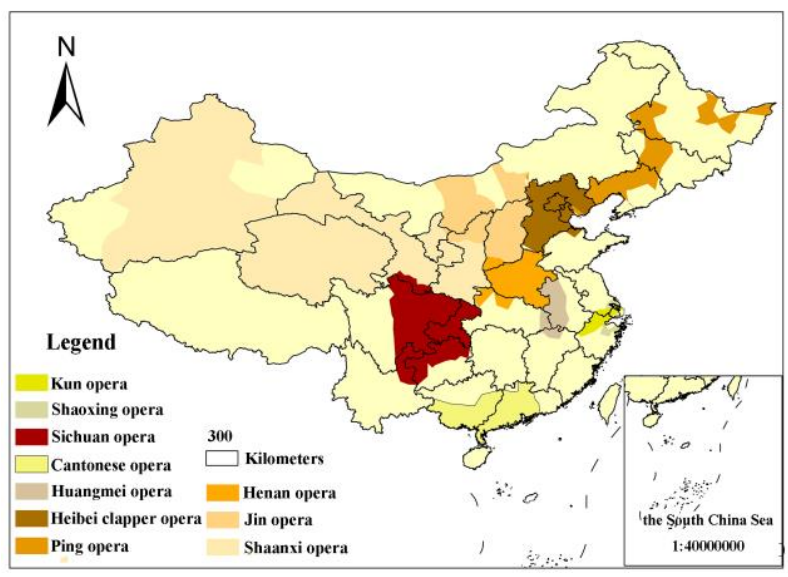

Fig. 3 Spatial distribution of opera

Generally speaking, the regions where these operas are popular and where they originate have consistency and difference in geographical background. Take the Shaanxi opera as an example. It originates from the 


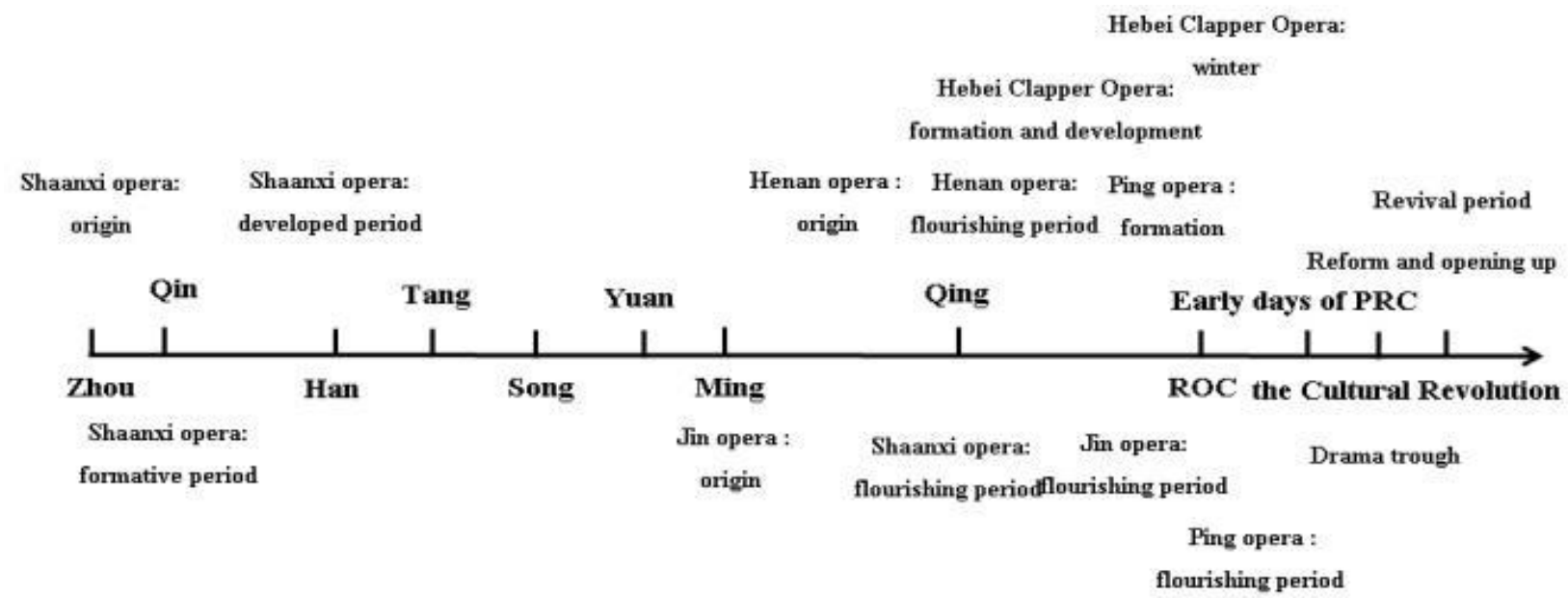

Fig .4 Development of Northern Chinese opera

temperate region, has a dry climate and obvious seasonality. Its economy and society are mainly agricultural, with relatively underdeveloped handicraft industry and backward commerce and transportation. But at the same time, there are great differences in ethnic culture and customs, so there is consistency and difference in geographical background. In addition, the relationship between the ten local operas can be divided by the oldest Shaanxi opera as the reference point: Henan Opera, Hebei clapper opera, Jin Opera, Ping opera represented by the direct influence of the Shaanxi opera. As well as Sichuan opera represented by the indirect influence of the Formation of the Shaanxi opera.

\subsection{Spatial and temporal differences of pronunciation and singing}

The ten local operas have gone through a long period of precipitation since their germination, and their pronunciation and singing show obvious heterogeneity in time and space. From the Qing Dynasty to the modern times, due to the poor stability of northern operas, except for Jin Operas, the pronunciation and singing of the other operas changed significantly, and the margin area was greatly reduced, while only the core area was retained. The Shaanxi opera and Hebei clapper opera changed the most (Fig.5). Southern dramas are more stable. The range of core area and edge area of Sichuan opera and Huangmei Opera changed slightly, while the distributionrange of Shaoxing opera was wider, which was obviously different from that of northern operas.

The northern operas originated early, dating back to the Zhou Dynasty (Fig. 4), but developed slowly after the Song Dynasty. Qing dynasty, the northern drama enter prosperous period, combined with the convergence of mandarin, Hebei clapper opera and Shaanxi opera flow in a wide range. Except for the three eastern provinces, other provinces have Shaanxi opera. The spread of Hebei clapper opera extends to some areas of Xinjiang and now Ulan Bator, Mongolia. The dialect area is inconsistent with the spatial distribution. After modern times, the locality of northern operas has been greatly weakened, and the singing style has undergone significant changes. In addition to the synchronous changes of Hebei clapper opera 's pronunciation and singing, the singing space of Shaanxi opera, Henan Opera and Ping opera has changed greatly. Among them, the singing style of Hebei clapper opera has undergone three different stages of development, from stable, smooth and concise, to sad, solemn and stirring, and then to loud, gorgeous and tasteful. The Shaanxi opera of Gansu province has not adopted the performance form of Qupai continuous patchwork, and the changes of plate style are more obvious, so there is an obvious regional shift in Shaanxi opera. In addition to the Shaanxi opera, the other five kinds of opera singing changes in stages. The founding of the People's Republic of China was an important time for the transformation of the singing styles of Henan Opera and Ping opera.

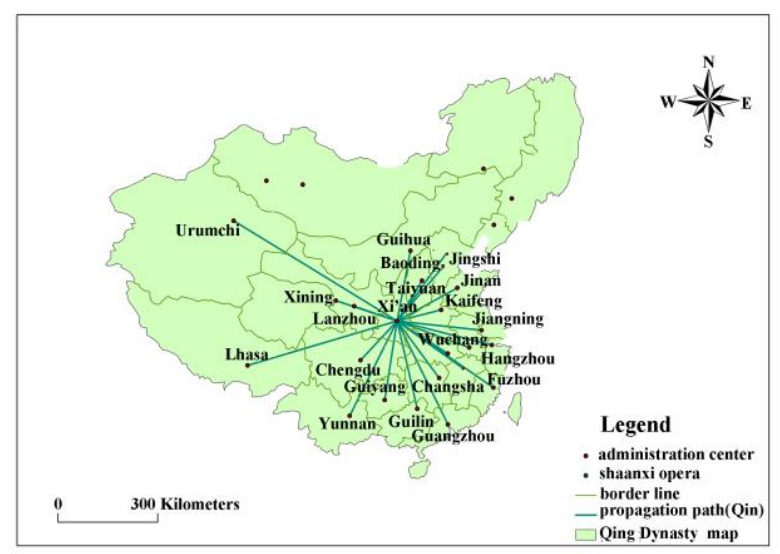



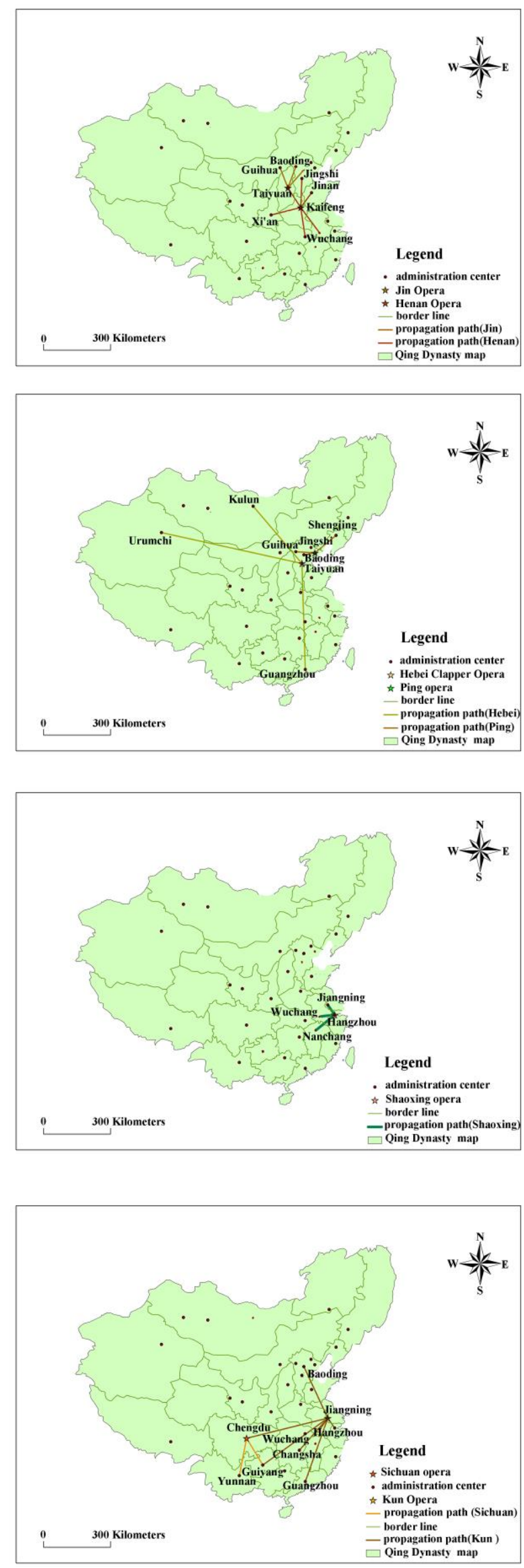

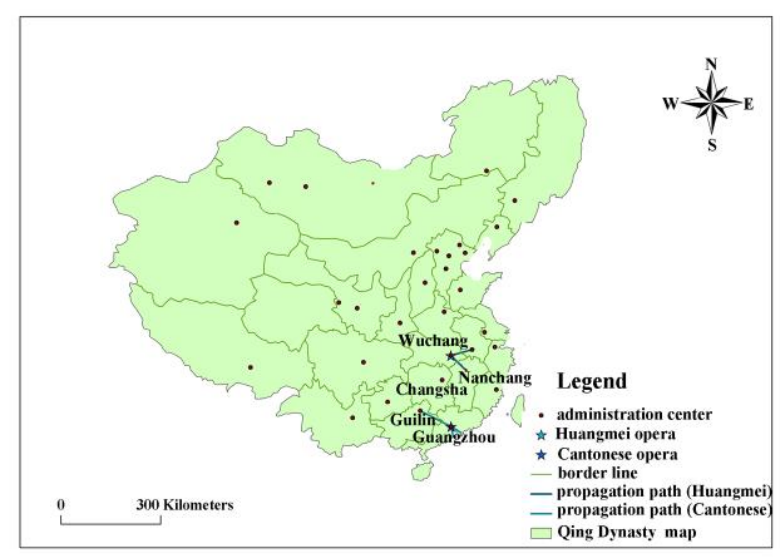

Fig. 5 Transmission scope of Operas in Qing Dynasty

The southern opera originated later and first appeared in the Tang Dynasty. However, after the Song Dynasty, southern operas developed more vigorously, and new operas were produced constantly. Most of them flourished in the Qing Dynasty, with Kun opera and Shaoxing opera being the most typical. Kun opera is distributed in Jiangsu, Anhui, Zhejiang, Beijing, Hunan, Sichuan, Guizhou, Guangdong and other provinces. The distribution of Cantonese opera has expanded to Hong Kong and Macao[17] (Fig.6 ). The core of opera is dialect. Except for Sichuan opera and Shaoxing Opera, the dialects of Huangmei Opera, Kun Opera and Cantonese all have obvious stage changes. Among them, Huangmei Opera and Cantonese Opera respectively realized the transformation from Huangmei mandarin to Anqing mandarin, and from central Plains to Cantonese. Kun opera, with the middle leaves of the Ming Dynasty as its boundary, experienced the transformation from multiple dialects to single dialect, but the regional and spatial distribution of dialects were roughly the same. The main component of opera is singing. Compared with the northern operas, the southern operas have less changes in singing. Except for the Shaoxing Opera, which responds to the development of The Times, the other operas have little changes in singing. 


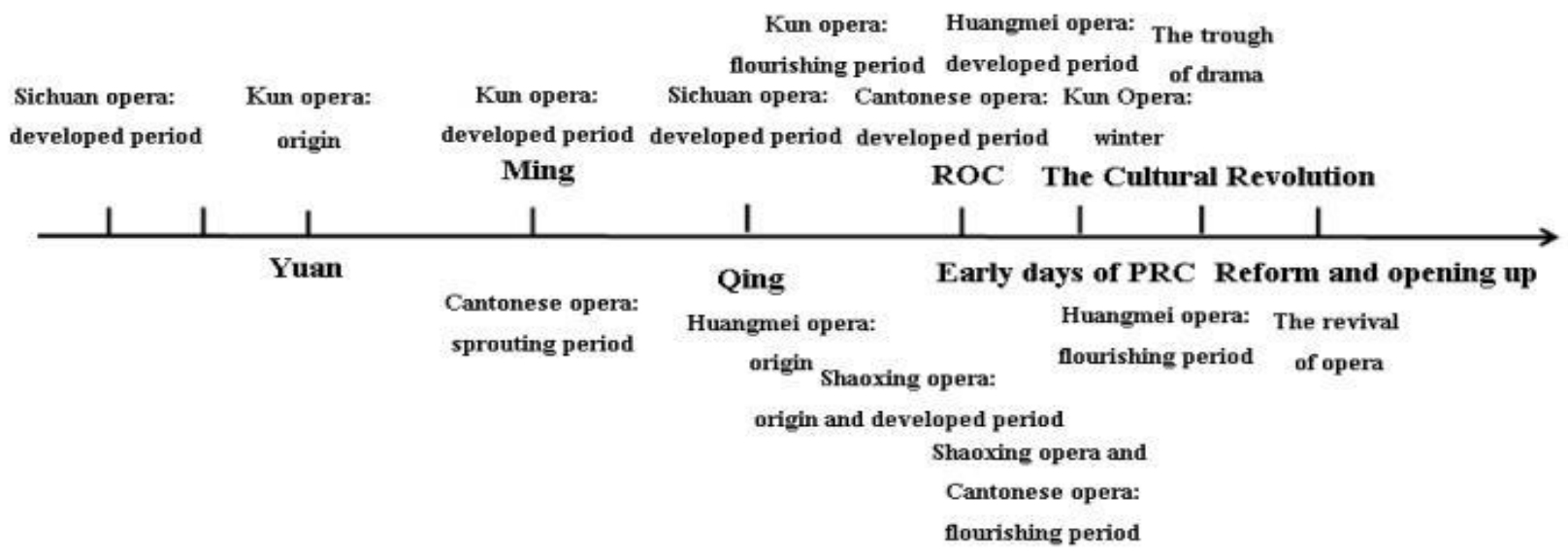

Fig .6 The development of Southern Chinese opera

\section{GEOGRAPHICAL MECHANISM ANALYSIS OF LOCAL DRAMA EVOLUTION}

\subsection{Factor role analysis}

By analyzing the changes of ten local operas, this paper analyzes the evolution mechanism of local operas from the perspective of new mobility paradigm. From the perspective of the motivation of spatial mobility, terrain profoundly affects the outward flow of opera and plays an important role in the diffusion of pronunciation and singing. Climate not only influences the local characteristics of opera pronunciation and singing, but also acts as an effective thrust for spatial flow. Economic development gathers financial and material resources, which is the continuous attraction and pull of spatial flow of drama. From the perspective of the mobility paradigm, the mobility is mainly derived from the daily communication generated from close proximity, that is, the results of communication brought by the decrease of distance.

During this process, the population flow, as the carrying and transmission media of the flow, spreads the opera culture to different spaces. Immobility has the relativity, is the regional characteristic changes relatively small performance. Among them, the mandatory policy factors control the trend of cultural values by changing the flow rights through the prism of time and space (constraints under specific time and space), and maintain the differences of opera to a certain extent. Dialect, as the foundation of regional emotional network, has strong vitality.

\subsection{Factor mechanism analysis}

\subsubsection{Motivation analysis - push}

\subsubsection{Climate}

Climate factor plays an important role in the formation of opera singing style[18-19]. It is an important factor that causes the artistic differences between southern and northern opera singing, and directly establishes the main melody of soft in the south and rigid in the north. Climate is also a catalyst for the outward flow of opera. The spatial distribution of Huangmei Opera is closely related to the climatic conditions. Huangmei county originated from Huangmei county, Hubei province, which is located in subtropical monsoon climate, with high annual precipitation and waterlogging. The root cause of the widespread distribution of Huangmei opera in Anhui province, Hubei province and Jiangxi province is that waterlogging promotes the spatial flow of people and materials.

\subsubsection{Terrain}

Terrain is the basic factor to shape the spatial pattern, and it has a limited and protective effect on opera. As a diffusion medium, liquidity carries and transmits the flow and transmission of other things[20]. Flat terrain or favorable water transport conditions provide conditions for the large-scale flow and diffusion of Shaanxi opera, Henan Opera, Ping Opera and Kun Opera, and limit the area of cultural diffusion in space.

\subsubsection{Motivation analysis - pull}

Economic factors. Economic development to a large extent determines the direction of drama flow. On the one hand, economic development has laid a solid material foundation for the growth and diffusion of local 
operas, which is mainly manifested in two aspects of economic attraction and market attraction. In the Tang Dynasty, the society was stable and rich, and the spiritual needs of the people were rich, which promoted the prosperity of Qinqiang opera in the Tang Dynasty. In the Southern Song Dynasty, the center of economic gravity moved south, and the southern economy began to surpass the northern economy. Kun Opera, Huangmei Opera and Shaoxing Opera drew nutrients from the southern region and developed rapidly. On the other hand, economic development is the continuous attraction of the spatial flow of drama. Various operas gather in economically developed areas, and the concentration degree of drama is highly coupled with economic development. Most major cities offer the area economy, wealth and resources, population and so on, occupies the absolute superiority of geography, not only gathered a large number of play groups, professional talent and folk performances, and the audience to large market, has broad market and considerable income, to attract artists to urban mobility, explore the new space for development.

\subsubsection{Mobility Paradigm}

\section{$\underline{\text { 3.2.3.1 Mobility Factor }}$}

Daily life. mobility has a background and context. By interweaving people, places, things and emotions, it forms the way the world works. Daily life, as the most extensive place for communication, can spread knowledge or information outward through people, things and things, providing places and other media for the flow of opera culture, and driving the development of opera.

Population mobility. The carrying and transmission of mobility cannot be separated from media. Artists and businessmen, as carriers of culture in spatial flow, bring regional cultural genes to new places, thus infecting new groups and directly leading to the formation of cultural enclaves from the original popular areas. Mobility can not only pass on art, but also convey emotion. The singing and recording of opera spread the culture contained in pronunciation to different spaces, and spread it in complex and rapid forms with the help of the flow of people and non-people. Beijing brings together the emotional exchange and resonance of people[21-24]。

\subsubsection{Immobility Factor}

Policy. As an ideology embedded in open political discourse, mobility will control and restrict the right to mobility with the help of policies. During the cultural revolution, the top ten local operas were constrained by the prism of time and space, resulting in the development of "low mobility", which was manifested in the rapid reduction of the core and marginal areas of operas and the serious compression of living space. It can be seen that there are social differences in spacetime compression, and the mobility after policy control will aggravate the differences in cultural development.

Dialect. Opera spreads in space with the help of human mobility, which depends on the established emotional network and common dialect foundation. Through the visual analysis of the spatial distribution of modern local operas and dialect areas (Fig.8), the popularity of Jin opera and other local operas has a high degree of coupling with the dialect areas. Influenced by the dialect landscape, opera endows opera with new locality under the local social norms, code of conduct and belief system through "mobile production" in similar dialect areas.

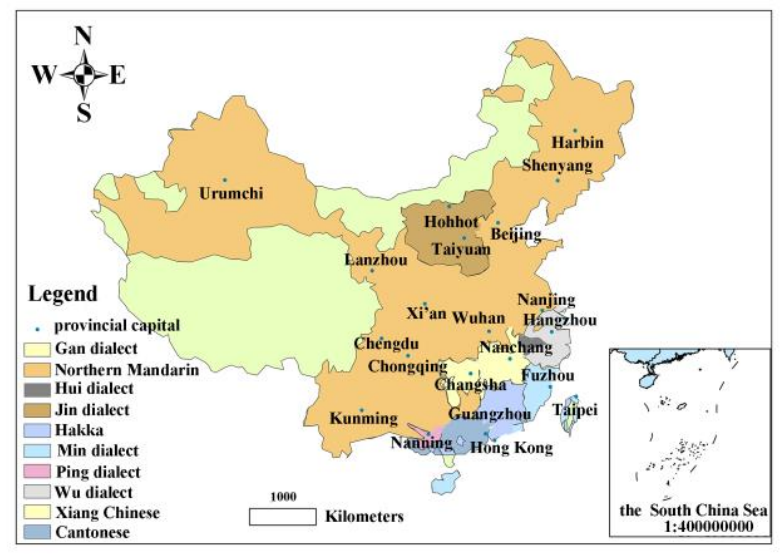

Fig. 7 Spatial distribution of ten dialects

\section{CONCLUSION}

This paper takes ten local operas as the research object, reveals the mechanism of regional differentiation from the perspective of mobility, and mainly draws the following two conclusions:

First, divided by the Qinling Mountains-Huaihe River line, northern operas form three different distribution structures linked by dialects. Among them, Henan opera, Jin opera and Hebei clapper opera are distributed in circles with the birthplace as the center, Shaanxi opera is mainly distributed in the west, and Ping opera is distributed in a double center structure. Although northern operas originated earlier and had a wider influence, their singing stability was poor and their cultural regions contracted seriously. Although Southern operas originated late, due to the influence of terrain, the strengthening effect of internal circulation is obvious, so the singing stability is high and the regional contraction is less. Among them, Huangmei Opera, Cantonese opera and Sichuan Opera are distributed in circles, while Shaoxing Opera and Kun Opera are distributed in dot structure. On the whole, influenced by many factors, more than half of the pronunciation and 
singing of opera have changed greatly, the dialect crisis is becoming more and more serious, and the marginal areas of the seven operas have decreased sharply. The living space of opera is becoming increasingly compressed, and the opera culture needs to be activated, inherited and protected urgently.

Second, in the perspective of the new mobility paradigm, the complex climate and flat terrain promote the short-distance outward spread of opera, while the prosperous economy is the attraction of driving the continuous outward flow of local opera. Taking immigration as the transmission medium, the opera culture is spread to different spaces through daily life; Through the constraints of specific time and space, maintain the core position of dialect in locality. Finally, a spatial flow system with economy as the pull, climate and terrain as the push, population flow, daily life as the media and policy protection dialect characteristics will be formed.

\section{REFERENCES}

[1] Yang Li-ming. On the language, singing tune and accompaniment of Huang mei Drama[J].Art Research,2018(01):58-59.

[2] Su Zhiyuan. The Musical Characteristics of Kunqu Opera singing tune-- On the whole song of The Hall of Eternal Life [J].Song of the Yellow River,2017(16):114-115.

[3] Zhang Yanli. On the influence of Jin zhong Dialect on the melody of Jin Opera[J]. Chinese Music,2012(02):189-192+205.

[4] Xia Bin. A Study on the Influence of Geographical environment on Huangmei Drama[J]. Yunnan Geographic Environment Research,2006(02):107110.

[5] He Zikun.The different flowers of Arbutus in different places-A study on the geographical environment of Cantonese opera's survival and reproduction[J]. Nan Guo Hong Dou,2009(04):7-8.

[6] Huang Hu. Review and Prospect of music geography research at home and abroad[J]. Journal of Xinghai Conservatory of Music, 2016(02):50-60.

[7] Zhang Jian, Wei Qian-ru, Rui Yang, et al. The Perception and identity of the cultural consumers to Qin opera: A case study of 'Yi Su She' and 'Shaanxi traditional opera institute'[J]. Human Geography, 2018,33(01):31-42.

[8] Ding Yan. A Discussion on the Relationship between Shaanxi Opera Culture and Geographical Environment based on the Perspective of cultural Geography[J]. Shanxi Agricultural Economy, 2017(08):20-21.
[9] Liu Qiang. Comparative Study on landscape Characteristics and Geographical influence factors of Shaoxing Opera and Shaanxi Opera[J].Tourism Overview,2015(10):295-296.

[10] Wu Feng. On the Cultivation of Henan Opera culture ecology from The Perspective of $\mathrm{Li}$ yuan Chun[D]. Nanjing University of the Arts,2012.

[11] Christaller W.1966 Central Places in Southern Germany.London:Prentice-Hall.

[12] Yang Xihao and Zhu Hong.2015.Progress and Revelation of Researches on the Angloophone Geography of mobilities.Journal of South China Normal University(Natural Science Edition), 47(2):1-11.

[13] Sun Jiuxia,Zhou Shangyi,Wang Nig,Zhu Hong,Zhou Daming,Zhen Feng,Liu Xinjian,Yang Jingjing,Chen Jingfu and Yang Xihao.2016.Mobility in Geographical Research:Time,Space And Society.Geographical Research,35(10):1801-1818.

[14] Aharon Kellerman. Mobility or mobilities: Terrestrial, virtual and aerial categories or entities?[J]. Journal of Transport Geography, 2010, 19(4) : 729-737.

[15] Adey P. Mobility[M]. London and New York: Routledge,2009.

[16] Guo Yongchang,Yao Huan. Spatial distribution and Formation mechanism of Chinese Placename Songs -- Based on the time section analysis of 19902015[J]. Journal of Anqing Normal University(Social Science Edition,2017,36(05):9297.

[17] Zhou Muchun. A study at the Evolution of Shaanxi Opera from Zhonglu Bangzi[D]. Shanxi Normal University,2016.

[18] Xia Fen. Culture Geography Research On Huangmei Drama[D]. Anhui Normal University, 2006.

[19] Hu Meijuan Ding Zhengshan Zhou Nianxing, et al. The spreading process and driving mechanism of Huangmei Drama culture[J].Cultural Industry Research,2018(02):252-267.

[20] Cai xiaomei,bu meiling and lijun.2020. See The World From A Mobile Perspective- A Review on "Mobility" Edited by Peter Adey. Tropical Geography,40(3) :455-465.

[21] Gu Lingsen. On the humanistic background of the appearance of Kun Opera[J]. Journal of Zhejiang Vocational Academy of Art,2009,7(01):42-49. 
[22] Wan Zhongru. "Guan Road", "Shang Road" and "Shen Road" -- A long-term, holistic view of Cantonese opera music transmission[J]. Chinese Music,2019(04):28-35.

[23] Zhang Chunjuan. Jin Merchants, immigrants and Opera[D]. Shanghai Theatre Academy,2013.

[24] Ma Da, Luo Dan. Generation Route and Survival Cause of Cantonese Opera Viewed from Music Geography[J]. Hundred Schools in Arts, 2017,33(03):168-174. 